EOMmun Communication et organisation

Or

Repenser la communication dans les organisations publiques

\title{
La communication par le bas au ministère de la Justice
}

\section{Carole Thomas}

\section{OpenEdition}

\section{Journals}

Édition électronique

URL : http://journals.openedition.org/communicationorganisation/810

DOI : 10.4000/communicationorganisation. 810

ISSN : 1775-3546

Éditeur

Presses universitaires de Bordeaux

Édition imprimée

Date de publication : 1 juin 2009

Pagination : 170-181

ISSN : $1168-5549$

Référence électronique

Carole Thomas, "La communication par le bas au ministère de la Justice », Communication et organisation [En ligne], 35 | 2009, mis en ligne le 01 décembre 2012, consulté le 30 avril 2019. URL http://journals.openedition.org/communicationorganisation/810; DOI : 10.4000/ communicationorganisation.810 
Dossier : Repenser la communication des organisations publiques

\section{Résumé}

$\mathrm{Au}$ travers d'une approche par les acteurs, cet article analyse le phénomène d'enrôlement de l'ensemble d'un ministère, celui de la Justice, dans les pratiques communicationnelles. Partant de la façon dont la communication s'est déconcentrée avec des dispositifs spécifiques, on a pu observer des changements opérationnels liés à l'arrivée de nouveaux acteurs, les magistrats, dans le processus de communication. Ces transformations sont porteuses d'effets en termes de relations avec les médias, facilitées par cette communication par le bas.

\section{Mots-clés}

Communicants, Communication gouvernementale, Justice, Magistrats, Médias.

\section{Abstract \\ The purpose is to analyse the operational changes connected with the decentralization of the communication functions in the ministry of Justice. So that we can explore how generating communication at the various levels of the institution can be analysed as a new process of communication of the public organizations. We also wonder about effects of the new organization on the press relations activities, which seem to be easier because based on a communication from the bottom.}

Key-Words

Communication actors, Governmental communication, Judges, Justice, Media.

Carole Thomas est Docteur en sociologie de l'ENS Cachan. Elle est responsable de la communication interne d'Immobilière 3F. 


\section{La communication par le bas au ministère de la Justice}

Carole Thomas

activcarole@yahoo.fr

Au-delà de la forme classique d'institutionnalisation de la fonction communication au sein de l'État [Ollivier-Yaniv, 1997], on constate, dans beaucoup de ministères, que les relations avec les journalistes deviennent constitutives de l'activité d'agents qui n'appartiennent à aucun service communication [Gimbert, 2007 : 346]. Paradoxalement cette forme de communication par le bas semble avoir une grande efficacité en termes de relations avec les journalistes, acteurs toujours prompts à chercher d'autres sources que les communicants [Legavre, 2007b]. C'est au développement de la déconcentration de la communication au sein du ministère de la Justice et à ses effets en termes de relations presse que nous allons nous intéresser dans cet $\operatorname{article}^{131}$. Nous verrons dans un premier temps que l'élargissement des fonctions communication s'explique par la nécessité, pour cette administration régalienne, de rendre des comptes. Revenir ensuite sur la division du travail de communication au sein du ministère nous permettra de voir émerger, aux côtés des communicants politiques et communicants publics, de nouveaux communicants, magistrats, et de comprendre en quoi leur position est une ressource dans les relations avec la presse.

\section{L'entrée du ministère de la Justice en communication : rendre des comptes}

Depuis la décennie 1990, les questions de justice sont fortement médiatisées et politisées. Cependant, la médiatisation qui a accompagné cette politisation, ne s'est pas installée «naturellement » au sein du ministère. La préoccupation d'ouverture aux médias est

\footnotetext{
${ }^{131}$ Le matériau empirique de cette recherche est constitué de propos d'acteurs de la communication du ministère de la Justice, de journalistes rubricards (Justice de la presse quotidienne nationale et régionale (50 entretiens)) ainsi que d'observations de situation de communication entre magistrats et journalistes au sein de l'Ecole nationale de la magistrature et de cours d'appel. Il fait partie de la thèse qui constitue la base de cet article, intitulée "Le bruit de la loi, comment les lois deviennent médiatiques", soutenue par l'auteur le 19 décembre 2008 à l'ENS Cachan sous la direction de Patrice Duran.
} 
d'abord le fruit des pressions exercées par les médias eux-mêmes pour «faire parler» la Justice. Certes, le procès d'Outreau a profondément marqué les magistrats et contribué à accélérer le processus communicationnel au sein du ministère. Mais les relations difficiles entre médias et magistrats ont des origines plus anciennes, liées aux nouvelles formes de médiatisation des faits divers à rebondissement [Garapon, 94], ou de scandales politico-judiciaires [Roussel, 2000] du début des années 1990. Ce ministère est d'ailleurs l'un des derniers à mettre en place un service d'information et de communication (SICOM) [Lévêque, 1993], en $1994^{132}$. La tradition de secret et de distance vis-à-vis des justiciables, et peut-être plus encore des journalistes, est une constante au sein de ce ministère [CivardRacinais, 2003]. Lorsque l'École nationale de la magistrature (ENM) a mis en place un module «Justice et médias » dans son programme de formation initiale en 1985 , les 150 jeunes auditeurs de justice se mettaient en grève au nom de l'indépendance de la justice. Aujourd'hui encore, on peut observer que la plupart des jeunes auditeurs de justice vivent mal ce module de formation.

Or, la médiatisation des grands procès et des réformes de la justice a placé le ministère de la Justice devant l'obligation de rendre des comptes [Vigour, 2006], ce qui constitue l'une des caractéristiques principales des transformations de l'action publique ces vingt dernières années. Le recours à la communication dans les ministères correspond en effet au "mouvement plus général de redéfinition des modes de justification du pouvoir politique» [Duran, 1999: 169]. Ainsi, les magistrats sont maintenant, eux aussi, amenés à rendre compte de leur action, pour communiquer sur un procès en cours ${ }^{133}$ ou pour expliciter, voire " défendre ", l'intérêt de certaines réformes de la justice. Il s'agit bien de mettre au jour leur action et de la justifier, non plus en interne, mais devant un «public » par l'intermédiaire des micros tendus par les journalistes. Concrètement, l'obligation de rendre compte de l'activité judiciaire «en temps réel » se décline sous la forme de dispositifs «d'urgence»: organisation de points presse, diffusion de communiqués, réponse dans les couloirs lors d'instructions.

Dans ce contexte, la place assignée à la communication, ainsi que son rôle au sein de l'institution judiciaire, se modifient profondément. La communication devient un devoir, une tâche nouvelle pour les

\footnotetext{
${ }^{132}$ Décret $N^{\circ} 94-188$ du 25 février 1994.

${ }^{133}$ Les procureurs de la République communiquent régulièrement avec la presse au cours d'un procès comme les y autorise l'article 11 du code de procédure pénale, alinéa 3 .
} 
magistrats, comprise comme la contrepartie de la responsabilité qu'ils exercent au nom de leurs missions régaliennes. La communication, ou encore la prise de parole en public et pour un public, constitue ainsi un enjeu institutionnel fort puisque, dans une forme de délégation, c'est le ministre, et plus largement le gouvernement, qui est engagé au travers de la parole de ces acteurs. Cela s'est traduit concrètement, au début des années 2000, par un élargissement de la fonction communication qui n'est plus cantonnée dans les mains de quelques professionnels du cabinet ou du SICOM comme le montre l'analyse de la division du travail de communication au sein du ministère.

\section{Les acteurs de la communication au ministère de la Justice}

La politisation de la justice s'est progressivement traduite par l'augmentation de l'offre d'information institutionnelle parallèlement aux efforts consentis par les procureurs dans les juridictions pour communiquer sur les affaires en cours. Cette nouvelle offre se caractérise par le recrutement ou la formation de personnes dédiées à la communication, que l'on peut classer en trois grandes catégories : les communicants politiques du cabinet, les communicants de l'administration centrale, les magistrats référents pour les relations presse lors des procès difficiles ou délégués à la communication dans les cours d'appel.

Très proches des acteurs politiques, les communicants politiques du cabinet [Legavre, 1994a] sont amenés à gérer la communication des ministres mais également celle du ministère. Leur statut est à la fois précaire, puisqu'ils sont le plus souvent conseillers techniques dans des cabinets ministériels éphémères, et en même temps très valorisant, devenant incontournables en interne comme en externe. Ils s'appuient le plus souvent sur les communicants politiques d'agences, les «spin doctors » [Esser et al., 2000], des experts en médias et relations publiques sollicités pour leur connaissance du marketing et des médias davantage que pour leurs connaissances politiques. Au sein du ministère de la Justice ils ont une trajectoire "classique» de communicant, étant passés par une formation généraliste et ayant une grande expérience des médias, mais peuvent aussi avoir été magistrat. La crédibilité d'un communicant cabinet réside dans son réseau de journalistes, dans sa capacité à pouvoir «faire parler de son ministre ».

Les communicants de l'administration centrale, qui travaillent au sein $\mathrm{du}$ Service central de l'information et de la communication (SCICOM), gèrent essentiellement la communication avec le grand public (dépliants d'information, campagnes de recrutement, journées 
portes ouvertes), la communication interne (site Intranet du ministère, éditions diverses) et des outils de communication pour le cabinet (études d'opinion, baromètre Justice, revues de presse). L'absence de relations avec les journalistes au sein de ce service constitue une vraie contrainte pour ces acteurs lorsqu'il s'agit d'asseoir leur crédibilité auprès des autres acteurs du ministère. En effet, le SCICOM n'est pas en capacité de mettre en place une stratégie de relations presse dans la mesure où le cabinet conserve cette prérogative, ou la sous-traite à certains services pour des lois particulières (justice de proximité médiatisée par la Mission justice de proximité) et des domaines d'actions spécifiques (Administration pénitentiaire, Protection judiciaire des mineurs). Cette distinction est emblématique de la division du travail de communication que l'on retrouve à l'échelle de l'Etat, avec la volonté de différencier les communicants politiques, évoluant au sein des cabinets et les communicants de l'administration affectés dans les SICOM. Chacun des deux types d'acteurs entretient le mythe d'une dichotomie traditionnelle politique/administration [Lavigne, 1997] qui semble pourtant dépassée tant les logiques de communication avec les médias ont été intégrées dans l'administration publique [Nollet, 2006].

D'autres acteurs de l'administration centrale entretiennent des relations avec les journalistes. Il s'agit des communicants des directions du ministère les plus exposées à la médiatisation: la direction de l'Administration pénitentiaire et la direction de la Protection judiciaire de la jeunesse. Le rôle de ces communicants consiste, notamment, à autoriser ou à susciter des reportages en prison ou dans des foyers de jeunes pris en charge par le ministère. Des correspondants relaient l'activité de ces communicants en région, notamment auprès de la Presse quotidienne régionale (PQR). Au niveau central et au niveau déconcentré, s'observe une distinction très clairement établie entre les acteurs " autorisés » à communiquer et les autres. Ainsi, les fonctionnaires (éducateurs, surveillants de prisons) ou magistrats de ces directions ne répondent pas directement aux sollicitations des journalistes, mais les renvoient sur la cellule de communication de la Direction qui fonctionne comme une gare de triage et de sélection des sollicitations, demandant à un tel de répondre aux questions, ou renvoyant vers le cabinet si la question est jugée «politique ». Ce partage des tâches très contrôlé, qui ne permet pas aux agents de ces directions de rentrer en contact direct avec des journalistes, est également présenté comme une stratégie visant à "protéger» les agents du ministère, les journalistes étant perçus comme des perturbateurs dans l'activité d'urgence. Reste que dans le 
cas du fonctionnement de la justice au quotidien, c'est-à-dire au sein des tribunaux, les magistrats sont amenés à rencontrer les journalistes pour parler des affaires en cours. Compte tenu de la difficile prévisibilité de cette communication, le ministère a tenté d'instituer un nouveau type de communicants, dont la principale caractéristique consiste à être magistrat.

Nouveaux acteurs de la communication, les magistrats référents ont été formés pour se consacrer aux relations presse dans le cas de «procès difficiles" ${ }^{134}$ en fonction de l'actualité judiciaire. La communication sur l'évolution des procès est considérée dans ce dispositif comme un élément contribuant directement à améliorer l'image de l'institution judiciaire. En tant qu'acteurs marginauxsécants [Crozier, 1977: 86] ces magistrats favorisent les relations entre les sphères médiatiques et judiciaires, par la connaissance et l'expérience qu'ils ont de leur métier de magistrat mais surtout la connaissance qu'ils ont des contraintes des journalistes. Ces derniers, a priori méfiants face à des personnes désignées par le ministère pour communiquer, se sont finalement laissé convaincre de l'intérêt de tels acteurs, à l'image du président de l'Association confraternelle de la presse judiciaire qui regroupe la plupart des rubricards Justice des médias français : "Au début, nous étions un peu dubitatifs. Nous pensions que cela servirait surtout à indiquer aux équipes de télé le meilleur endroit pour faire leur compte-rendu du soir, où se trouvaient les toilettes et l'heure... En fait, ce n'est pas vrai. Dans l'ensemble, ils font bien ce qu'ils ont à faire. Ils préparent les accréditations. Ils relaient les messages. Ce n'est pas mal $\gg^{135}$.

Ainsi, parmi les acteurs qui ont porté le changement dans le sens d'une plus grande ouverture aux stratégies de rapprochement avec les journalistes, politique qualifiée de "plus offensive», ces magistrats ont joué un rôle important. Ayant rapidement assis leur légitimité auprès des journalistes, ils ont pu, du même coup, valoriser leur rôle au sein du ministère. Pour ce nouveau groupe d'acteurs, le " passage » par la communication a constitué un véritable tremplin en termes de carrière, leur apportant à la fois une compétence complémentaire et une forme de reconnaissance inédite auprès de leurs pairs. Ils viennent

134 Il s'agit en fait des procès à fort risque médiatique compte tenu, notamment, de la personnalité de l'accusé, de la nature du crime ou des caractéristiques des victimes. Le premier procès concerné fut celui d'Emile Louis à Auxerre en 2004, puis celui d'un réseau de pédophilie à Angers en 2005.

${ }^{135}$ Entretien du 29 août 2007. 
par exemple former les jeunes auditeurs de justice aux relations avec les médias à l'Ecole nationale de la magistrature de Bordeaux. Par ailleurs, le premier à avoir exercé ces fonctions lors du procès d'Emile Louis à Auxerre en 2004 est devenu chef du SCICOM deux ans plus tard. D'autres magistrats sont entrés dans les fonctions de communication depuis 2003: les Magistrats délégués à la communication (MDC). Ils sont nommés au sein de chaque cour d'appel pour relayer les actions du SCICOM en région. Ils mettent en place des stratégies de communication locale, visant essentiellement à rendre plus crédibles et plus visibles les efforts de l'institution pour s'ouvrir au public. Ils disposent de moyens restreints en personnel et en financements. Cependant, l'émergence de cette fonction est très intéressante en ce qu'elle laisse finalement une grande marge de manœuvre aux acteurs. Certains s'en sont spontanément saisis pour établir des relations avec les médias de façon très régulière comme nous allons maintenant le voir.

\section{Communiquer sans être communicant, une ressource dans l'interaction avec les journalistes}

De plus en plus de MDC profitent de l'ouverture à la communication de leur ministère pour agir localement. Ainsi, ils prennent des initiatives pour expliquer aux journalistes les réformes, les nouveaux dispositifs judiciaires, les bilans d'activité et, par ce relais, tentent de communiquer vers le grand public. Certains magistrats délégués à la communication mettent en place des événements spécifiques pour les médias. Nous nous arrêtons ici sur l'exemple d'un petit déjeuner presse mensuel organisé par le MDC de la cour d'appel d'Aix en Provence. Rompu à l'exercice, il lance le débat ${ }^{136}$ entre les journalistes et les experts qu'il a conviés pour répondre à leurs questions ainsi : "Donc je vous propose d'aborder le thème de l'application de l'exécution des peines sous un angle très pratique. Il ne s'agit pas ici de faire un cours de droit ou de délivrer un message très théorique. Ce sont des praticiens qui sont autour de la table. Cela permet peutêtre de développer la communication sur ce qui se passe "après", ce qui est essentiel, car à quoi servent les peines si elles ne sont pas ou mal exécutées?» Lors de ce petit déjeuner, les principaux médias régionaux sont représentés autour de la table, avec deux journalistes de La Provence, un de Var Matin et un de France 3 Provence. Pour exposer les nouveaux dispositifs dont il est question et répondre aux

${ }^{136}$ Il s'agit ici d'un petit déjeuner presse, auquel nous avons assisté, portant sur la réforme de l'exécution des peines, organisé par le MDC de la cour d'appel d'Aix en Provence, le 28 mars 2007. 
sollicitations des journalistes, plusieurs interlocuteurs sont réunis : un juge d'application des peines, un avocat général et la chargée de communication régionale de l'Administration pénitentiaire. Un peu plus tard, le procureur général près la cour d'appel rejoindra le groupe, marquant l'importance qu'il accorde à la rencontre.

Le développement de réunions de cette nature avec la presse est perçu comme "chronophage » mais productif, voire valorisant pour celui qui l'organise. Ce sont des moments où la communication en face à face implique à la fois une forte réactivité, une bonne maîtrise technique des dossiers ainsi qu'une posture relationnelle adéquate. L'objectif est de créer une relation de confiance avec ces journalistes et d'éviter, à moyen terme, qu'ils parlent de manière négative de l'action de l'administration judiciaire. Il s'agit de donner des informations et simultanément de fournir des clés de lecture des réformes : la relation de confiance avec les journalistes devient alors une ressource précieuse puisqu'elle offre la possibilité aux magistrats de la cour d'appel, dans une certaine mesure, de guider l'interprétation que les journalistes peuvent faire des documents transmis. Ce type de rencontre peut être analysé à la fois comme une entreprise de justification de l'action publique et comme une réunion « technique ». D'une part, l'explicitation de l'action menée par le ministère suppose, pour ces magistrats, de la présenter en la défendant. D'autre part, la communication d'informations plus pratiques s'appuie sur des rapports techniques, documents internes, non produits spécifiquement pour la presse.

Cette caractéristique, liée aux contingences matérielles, en particulier au fait que le MDC dont il est question ici cumule cette fonction avec celle de secrétaire général de la cour d'appel et dispose de peu de temps pour réaliser des documents dédiés aux médias, rend finalement la démarche encore plus crédible aux yeux des journalistes. Le souci de donner à voir du «concret» lors de ce petit déjeuner va jusqu'à la démonstration matérielle. Le bracelet électronique et tous ses attributs passent ainsi de main en main autour de la table, circulation accompagnée de tentatives d'humour embarrassées sur son "look branché ».

Pensée comme un dispositif de protection contre le risque médiatique, la communication nous est présentée par ce MDC comme faisant partie intégrante du travail de l'administration. Cette appropriation de l'enjeu montre comment cette forme d'apprentissage collectif de la fonction communication a finalement irrigué tout un système qui se met en ordre de communiquer pour rendre des comptes sur son 
activité. Pour ce magistrat, produire la réforme ne suffit pas. Le rôle des agents du ministère consiste aussi à la publiciser. Cette démarche facilite les relations avec les journalistes qui trouvent ainsi des sources moins instituées, bienveillantes à leur égard et susceptibles d'être plus « authentiques », plus directes dans leurs messages.

- "C'est beaucoup plus facile de travailler, pour nous journalistes, avec des magistrats, que de travailler avec tous les autres communicants qui, eux, sont des filtres. J'ai un accès direct aux magistrats. Je peux aller voir le procureur et les autres magistrats. Il y a une relation entre nous et il y a un accès direct. Je ne passe pas par vingt-cinq boîtes de communication qui vont me dire qu'aujourd'hui elles ne communiquent pas parce que cela ne les arrange pas.

- Des filtres, c'est-à-dire?

- C'est le verrouillage complet. Quand vous avez le ministre, c'est la langue de bois. Il n'y a pas grand-chose d'intéressant. Ils essaient de nous refourguer une interview quand ils font un déplacement en province $»^{137}$.

Ce journaliste explique par ailleurs comment il est entré dans une relation d'échange et écrit volontairement des papiers en phase avec les attentes de ce MDC qui lui facilite la vie en lui amenant de la " vraie » matière première lors de ces petits déjeuners : "Ils font cet effort de communication, ce serait dommage de ne pas renvoyer l'ascenseur. En général, ils sont contents. Ce que nous avons fait sur les tutelles [ $1^{\text {er }}$ petit déjeuner] a satisfait tout le monde. Le lecteur est intéressé. C'est à nous de puiser, dans cette matière première, quelque chose ». Les deux mondes, a priori si opposés, celui des magistrats et celui de la communication et de la presse, sont ici rapprochés pour former une sorte d'idéal du communicant Justice. Un idéal dans lequel se projette toujours davantage de magistrats, et plus seulement ceux qui ont été désignés pour communiquer. En effet, depuis 2003, les 181 procureurs de la République et les 35 procureurs généraux ont tous participé à des séances de média-training d'une journée, leur permettant de s'exprimer " face caméra ». "Plus de 90 $\%$ des procureurs de la République étaient satisfaits des sessions de média-training. Ils demandaient, dans la même proportion, le renouvellement des formations. Après le temps incontournable de

${ }^{137}$ Entretien du 28 mars 2007 avec le rubricard Justice du journal La Provence. 
réserve et de distance, cela a plutôt pris et même bien pris ${ }^{138}$. Selon l'appel d'offres lancé par le ministère pour le montage de ces formations, il s'agit d'abord d'apprendre à « maîtriser son discours" ; ensuite d'occuper le terrain et d'éviter que d'autres ministères n'aient la part belle dans les médias sur les affaires de délinquance ou les affaires criminelles. En effet, les médias donnent à voir la concurrence permanente qui se joue entre le ministère de l'Intérieur et le ministère de la Justice sur ces sujets.

Ces deux éléments constituent des raisons explicites pour le cabinet de former les magistrats à la communication comme le souligne une communicante cabinet: "La seule chose dont je suis fière, c'est d'avoir poussé Perben [ministre de la Justice entre 2002 et 2005] et la chef du SCICOM à faire de la formation des magistrats à la prise de parole. J'en avais assez de voir à la télé, dès qu'il y avait une affaire, des flics. On a appris aux préfets et aux flics à parler et les magistrats ne savaient pas s'exprimer. Ils s'exprimaient rarement ou mal, à part quelques-uns qui avaient du talent. Lors d'une enquête, il fallait avoir le procureur de la République, ou le substitut du procureur, à la télévision et non plus les flics ».

Longtemps encadrée et assurée exclusivement de manière centrale au niveau de la chancellerie, la communication du ministère de la Justice s'est donc déconcentrée dans les tribunaux depuis quelques années. Après un premier mouvement d'institutionnalisation de magistrats communicants, la fonction communication poursuit sa « démocratisation", dans un processus d'appropriation des principes de base des relations avec les journalistes. Convaincus de la nécessité d'ouvrir l'institution vers l'extérieur, les magistrats se trouvent de plus en plus légitimes à expliquer les décisions de justice, à apporter un éclairage pédagogique sur la loi, son application, les futures réformes.

La logique d'efficacité dans les relations avec les médias, qui suppose une plus grande marge de liberté pour les acteurs, afin que puisse se mettre en place des relations de confiance avec les journalistes, trouve ici un aboutissement. Par les formations qu'elle organise ou la liberté qu'elle laisse aux acteurs qui souhaitent communiquer, l'institution joue donc elle-même la carte de la communication par le bas, consciente de son impact sur les relations avec les journalistes même si cette communication comporte des risques liés à la diversité des pratiques sur le terrain. La communication publique est ainsi tiraillée aujourd'hui entre sa logique d'institutionnalisation et de contrôle qui a

\footnotetext{
${ }^{138}$ Entretien du 11 juillet 2006 avec la responsable du SCICOM.
} 
pu produire un certain nombre de cloisonnement de la fonction, et sa logique d'efficacité, qui l'incite à donner un rôle de communicant à des agents qui n'en avaient pas jusqu'ici.

\section{Bibliographie}

CIVARD-RACINAIS A., Le journaliste, l'avocat et le juge, Paris, L'Harmattan, 2003, 337 p.

CROZIER M., FRIEDBERG E., L'acteur et le système, Paris, Seuil, $1977,500 \mathrm{p}$.

DURAN P., Penser l'action publique. Paris, LGDJ, 1999, 216 p.

ESSER F., REINEMANN C., FAN D., "Spin doctors in British and German election campaigns : how the press is being confronted with a new quality of political PR", European Journal of Communication, 2000, vol. 15 , pp. 209-39.

GARAPON A., «La Justice est-elle "délocalisable" dans les médias ?» Droit et Société, 1994, vol. 26, pp. 73-89.

GIMBERT V. L'Etat sanitaire en question. Les administrations à l'épreuve des risques. Thèse de doctorat en Sociologie : École normale supérieure de Cachan, 2006, 499 p.

LAVIGNE A., «La communication gouvernementale à l'ère de la gestion de type entrepreneurial et de la médiatisation de la politique : quelques repères théoriques », In Quaderni, 1997, vol.33, pp.119-30.

LEGAVRE J-B., Conseiller en communication politique. L'institutionnalisation d'un rôle. Thèse de doctorat en Science politique : Université Paris I-Sorbonne, 1993a, 736 p.

LEGAVRE J-B., "Je t'aime moi non plus". Les relations d'"associésrivaux" entre journalistes et communicants. Mémoire pour l'habilitation à diriger des recherches en sciences de l'information et de la communication : Université de Versailles Saint-Quentin-enYvelines, 2007b, $524 \mathrm{p}$.

LÉVÊQUE S., «L'invention de la communication : la mise en place d'un SICOM au ministère de la Solidarité, de la Santé et de la protection sociale en 1990 », In Revue française des affaires sociales, 1993; vol.3, pp. 137-49.

NOLLET J., «Les communicateurs de ministère entre champs bureaucratique et journalistique ", Chupin I, et Nollet J. (dirs). Journalisme et dépendances. Paris : L'Harmattan, 2006, pp.161-85. 
La communication par le bas au ministère de la justice

OLLIVIER-YANIV C., Etat et communication gouvernementale : histoire d'une nécessité démocratique. Thèse de doctorat en Science politique : Université de Paris I - Sorbonne, 1997, 624 p.

VIGOUR C., «Justice : l'introduction d'une rationalité managériale comme euphémisation des enjeux politiques »,In Droit et Société, 2006, vol. 63, pp. 425-55. 